\title{
Rastreio de dislipidemias em crianças e adolescentes - a evidência que sustenta as recomendações
}

Marisa Loio*, Daniela de Andrade Maia*

\section{RESUMO}

O rastreio de dislipidemias em crianças e adolescentes tem sido recentemente tema de orientações clínicas emitidas por diversas sociedades médicas, incluindo a Direção-Geral da Saúde em junho de 2013.

Estas recomendações surgiram num contexto atual de aumento da prevalência da obesidade e excesso de peso em crianças. Além disso, estudos demonstram que o processo aterosclerótico pode ter início na infância e que há uma tendência para as crianças com dislipidemias manterem o perfil lipídico alterado em idade adulta. Contudo, questiona-se se o rastreio de dislipidemias em crianças está associado a diminuição do risco e da morbimortalidade cardiovasculares.

O presente artigo visa rever as diferentes estratégias de rastreio propostas pelas principais sociedades médicas e analisar a evidência que sustenta estas recomendações.

As normas de orientação que recomendam o rastreio de dislipidemias não se baseiam em estudos que comprovem o benefício do mesmo na diminuição do risco e da morbimortalidade cardiovasculares, pelo que a necessidade da realização do rastreio deve ser ponderada individualmente.

Palavras-chave: Dislipidemias; Rastreio; Criança; Adolescente.

\section{A RECOMENDAÇÃO DA DIREÇÃO-GERAL DA SAÚDE}

A s dislipidemias constituem fator de risco estabelecido para desenvolvimento da doença cardiovascular (CV), principal causa de morte em Portugal em adultos. Existe cada vez mais evidência de que o processo aterosclerótico tem início em idade pediátrica, ${ }^{1}$ processo este que se desenvolve em relação com a presença e intensidade de conhecidos fatores de risco $\mathrm{CV}$, nomeadamente de dislipidemias. Estes factos, associados ao aumento da prevalência de obesidade e excesso de peso na infância, ${ }^{2-3}$ têm conduzido ao desenvolvimento recente de orientações clínicas relativamente ao rastreio de dislipidemias em crianças e adolescentes.

Em junho de 2013, a Direção-Geral da Saúde (DGS) emitiu a norma de orientação clínica referente ao Pro-

*Médica interna de Medicina Geral e Familiar, USF Santa Clara, ACES Grande Porto IV grama Nacional de Saúde Infantil e Juvenil (PNSIJ), a qual passou a fornecer recomendações relativas ao rastreio de dislipidemias em idade pediátrica. ${ }^{4}$

A DGS recomenda o rastreio de dislipidemias entre os 2 e os 4 anos de idade se existirem antecedentes familiares (primeiro e segundo grau) de doença CV precoce ou perfil lipídico alterado. ${ }^{4}$ É considerada doença CV precoce a ocorrência de angor ou enfarte agudo do miocárdio, acidente vascular cerebral, doença arterial periférica e morte súbita antes dos 65 anos no género feminino e antes dos 55 anos no género masculino. ${ }^{4} \mathrm{~A}$ alteração do perfil lipídico é definida como: colesterol total (CT) superior a $240 \mathrm{mg} / \mathrm{dl} \mathrm{e} / \mathrm{ou}$ colesterol LDL (LDL-c) superior a $130 \mathrm{mg} / \mathrm{dl}$ e/ou triglicerídeos (TG) superiores a $170 \mathrm{mg} / \mathrm{dl}$ e/ou colesterol HDL (HDL-c) inferior a $35 \mathrm{mg} / \mathrm{dl} .{ }^{4}$ É também preconizado o rastreio de qualquer criança ou adolescente com antecedentes pessoais de excesso de peso, obesidade, diabetes mellitus, hipertensão arterial, doenças renais, doenças car- 
díacas, doenças hormonais e/ou de metabolismo e, ainda, com terapêuticas prolongadas com fármacos hiperlipidemiantes (corticoides, antidepressivos, anticonvulsivantes, betabloqueadores, tuberculostáticos, contracetivos orais, derivados do ácido retinóico e antirretrovirais). ${ }^{4}$ Quando não estão presentes os critérios anteriores, a DGS recomenda que o rastreio seja realizado de forma oportunística (acima dos 2 anos de idade), ou seja, aquando da necessidade de efetuar colheita de sangue com fins diagnósticos no período pré-operatório ou em vigilância de rotina. ${ }^{4}$

A maioria das crianças com dislipidemias apresenta uma forma idiopática (poligénica, associada a fatores de risco ou multifatorial), enquanto uma minoria apresenta a forma monogénica, como a hipercolesterolémia familiar ou secundária a outras causas específicas que alteram o metabolismo das lipoproteinas. ${ }^{5}$ As formas secundárias estão associadas a uma grande variedade de condições, nomeadamente fármacos, distúrbios endócrinos (hipotiroidismo, diabetes mellitus), infeções, doenças renais, inflamatórias crónicas, hepáticas, entre outras. ${ }^{1}$

À luz do conhecimento atual, segundo vários estudos de coorte, parece haver uma tendência para manter o mesmo padrão de valores de CT e LDL-c desde a infância até à idade adulta, sendo que cerca de $50 \%$ das crianças que têm níveis de lípidos acima do percentil 75 na infância terão níveis de lípidos também elevados na idade adulta. ${ }^{1}$ Além disso, vários estudos patológicos de autópsia realizados em crianças, adolescentes e jovens adultos mostraram uma correlação significativa entre os níveis de lípidos e a deposição de gordura arterial, o aparecimento de lesões precoces de aterosclerose (estrias lipídicas), bem como a sua subsequente progressão com a idade. ${ }^{1,5}$ Também segundo estudos de imagem não-invasivos, níveis anormais de lípidos na infância associam-se a alterações da parede arterial consideradas precursoras de aterosclerose avançada. ${ }^{1}$ No entanto, apesar destes dados e de algumas das atuais recomendações das principais sociedades médicas no que diz respeito ao rastreio de dislipidemias em idade pediátrica, na prática clínica questiona-se se este procedimento está associado à diminuição do risco e da morbimortalidade CV.

\section{RECOMENDAÇÕES INTERNACIONAIS}

As orientações para o rastreio de dislipidemias em idade pediátrica diferem entre as várias sociedades médicas, nomeadamente em relação à indicação para realização de rastreio, à idade alvo e aos métodos de rastreio.

A United States Preventive Services Task Force (USPSTF) e o Institute for Clinical Systems Improvements (ICSI) não recomendam a favor ou contra o rastreio em idade pediátrica ${ }^{6-7}$ e a Royal Australian College of General Practitioners (RACGP) apenas recomenda o rastreio nos adultos. ${ }^{8}$

Por outro lado, o National Heart, Lung, and Blood Institute (NHLBI) e a American Academy of Pediatrics (AAP) recomendam o rastreio universal em determinadas faixas etárias (9-11 anos; 17-21 anos) por considerarem que não existe um marcador pessoal ou familiar que comprovadamente permita a seleção de crianças com risco aumentado de dislipidemias. ${ }^{1,9}$

Tal como a DGS, a American College of Obstetricians and Gynecologists (ACOG), a American Association of Clinical Endocrinologists (AACE) e a American HeartAssociation (AHA) assumem uma posição intermédia, recomendando o rastreio de dislipidemias apenas em crianças com fatores de risco. ${ }^{4,10-12}$ Os fatores de risco considerados pelas sociedades médicas supracitadas diferem entre si. Parece reunir maior consenso a seleção de crianças e adolescentes com história familiar de dislipidemias ou doença $\mathrm{CV}$ precoce (considerada antes dos 55 anos no sexo masculino e antes dos 65 anos no sexo feminino); história familiar desconhecida; crianças com outros fatores de risco, nomeadamente obesidade, diabetes mellitus ou hipertensão arterial. Também o intervalo etário preferencial para a determinação do perfil lipídico não é uniforme. A DGS é aquela que recomenda o intervalo mais estreito (2-4 anos) para a realização do rastreio, não tendo sido encontrada qualquer evidência que fundamente esta recomendação. ${ }^{4} \mathrm{~A}$ DGS é a única a recomendar o rastreio oportunista a partir dos 2 anos de idade. ${ }^{4}$

\section{EM QUE SE BASEIAM ESTAS RECOMENDAÇÕES?}

As normas de orientação que recomendam o rastreio de dislipidemias não se baseiam em estudos que comprovem o benefício do mesmo na diminuição do risco e da morbimortalidade CV. Consistem em consensos de peritos, baseados no facto de a alteração do perfil lipídico em idade pediátrica conduzir ao apare- 
cimento de lesões de aterosclerose nas paredes vasculares $^{1,5} \mathrm{e}$ de existir uma tendência para as crianças com dislipidemias manterem o perfil lipídico alterado na idade adulta. ${ }^{1}$ Assim, as recomendações a favor do rastreio de dislipidemias em idade pediátrica são construídas a partir destas evidências e inferem que a deteção e intervenção precoces poderão alterar o prognóstico em termos de risco e morbimortalidade CV.

Haney et $\mathrm{al}^{5}$ procuraram avaliar o benefício do rastreio de dislipidemias em crianças e adolescentes no atraso do aparecimento ou na redução da incidência de eventos coronários na idade adulta. Apesar de se tratar de uma revisão sistemática de boa qualidade, utilizando uma pesquisa criteriosa e seguindo a metodologia específica utilizada pela USPSTF nas suas revisões, não foram encontrados quaisquer estudos que avaliassem o efeito do rastreio de dislipidemias em idade pediátrica nos níveis lipídicos em idade adulta ou na morbimortalidade de causa $\mathrm{CV} .{ }^{5}$

Após realização de uma pesquisa nas principais bases de dados científicas, constata-se que não existem estudos que avaliem o benefício do rastreio em melhorar os marcadores de risco CV (disease-oriented-outcomes) ou em protelar ou diminuir a incidência de doença CV na idade adulta (patient-oriented-outcomes). Neste contexto, permanece a dúvida se a realização do rastreio em idade pediátrica tem impacto na diminuição do risco e da morbimortalidade $\mathrm{CV}$.

Para além da ausência de evidência relativamente ao benefício do rastreio, também não existem estudos que avaliem o efeito do tratamento em crianças com dislipidemia idiopática e secundária (maioria dos casos). Apenas em crianças com dislipidemia monogénica, a intervenção dietética/farmacológica parece melhorar o perfil lipídico e os marcadores imagiológicos de aterosclerose. ${ }^{1}$

\section{POTENCIAIS RISCOS DO RASTREIO}

A decisão de submeter ou não as crianças e adolescentes a rastreio deve ter em consideração o balanço entre os potenciais benefícios e danos inerentes a este procedimento. Apesar de não existirem estudos que o comprovem, ${ }^{5}$ o rastreio de dislipidemias em idade pediátrica pode ser um fator de ansiedade nas crianças e familiares, quer relacionado com o procedimento em si (picada de agulha, necessidade de jejum), quer asso- ciado a um resultado anormal. Além disso, a presença de um resultado normal pode ser um perpetuador de comportamentos de estilo de vida desadequados. Outra questão a ter em consideração, quando o rastreio for positivo para dislipidemias, é a relação benefício/risco do tratamento farmacológico em idade pediátrica. Atualmente existem estudos que demonstram que as estatinas são efetivas e habitualmente bem toleradas. No entanto, há relatos de efeitos adversos associados ao tratamento e são necessários estudos que comprovem a sua segurança a longo prazo, principalmente nas crianças com hipercolesterolemia primária que irão realizar tratamento farmacológico ao longo da vida. ${ }^{13}$

\section{DISCUSSÃO}

Recentemente várias sociedades médicas elaboraram orientações clínicas relativas ao rastreio de dislipidemias em crianças e adolescentes. Segundo uma perspetiva de prevenção quaternária, o médico de família é confrontado com a dúvida se o benefício supera os riscos inerentes a esta intervenção. De facto, trata-se de um procedimento invasivo com potenciais implicações futuras no bem-estar da criança e da família.

A pesquisa em bases de dados científicas permite concluir que não existe evidência do benefício do rastreio de dislipidemias em crianças e adolescentes na diminuição do risco e da morbimortalidade CV.

É importante a realização de estudos metodologicamente robustos e orientados para o doente que avaliem os potenciais benefícios/riscos do rastreio e subsequente intervenção terapêutica, de forma a permitir uma prática clínica mais alicerçada na evidência científica. Neste contexto, considera-se também pertinente a revisão das orientações que constam no atual PNSIJ da DGS.

\section{REFERÊNCIAS BIBLIOGRÁFICAS}

1. National Heart, Lung, and Blood Institute. Expert Panel on integrated guidelines for cardiovascular health and risk reduction in children and adolescents. U.S. Department of Health and Human Services; 2011.

2. Ogden $\mathrm{CL}$, Carroll MD, Kit BK, Flegal KM. Prevalence of obesity and trends in body mass index among US children and adolescents, 19992010. JAMA. 2012;307(5):483-90.

3. National Center for Health Statistics. Health, United States, 2011: with special features on socioeconomic status and health. Hyattsville, MD: U.S. Department of Health and Human Services; 2012.

4. Direção-Geral da Saúde. Programa nacional de saúde infantil e juvenil. Lisboa: DGS; 2013. 
5. Haney EM, Huffman LH, Bougatsos C, Freeman M, Fu R, Steiner RD, et al. Screening for lipid disorders in children and adolescents: systematic evidence review for the US Preventive Services Task Force. Rockville, MD: Agency for Healthcare Research and Quality; 2007.

6. US Preventive Services Task Force. Screening for lipid disorders in children: US Preventive Services Task Force Recommendation Statement. Pediatrics. 2007;120(1):189-99.

7. Wilkinson J, Bass C, Diem S, Gravley A, Harvey L, Maciosek M, et al. Preventive services for children and adolescents. Bloomington (MN): Institute for Clinical Systems Improvement; 2012.

8. Royal Australian College of General Practitioners. Guidelines for preventive activities in general practice. 8th ed. East Melbourne: Royal Australian College of General Practitioners; 2012.

9. Expert Panel on Integrated Guidelines for Cardiovascular Health and Risk Reduction in Children and Adolescents, National Heart, Lung, and Blood Institute. Expert Panel on integrated guidelines for cardiovascular health and risk reduction in children and adolescents: summary report. Pediatrics. 2011;128 Suppl 5:S213-56.

10. American College of Obstetricians and Gynecologists. Guidelines for adolescent health care. 2nd ed. American College of Obstetricians and Gynecologists Committee on Adolescent Health Care; 2011.

11. Jellinger PS, Smith DA, Mehta AE, Ganda O, Handelsman Y, Rodbard HW. American Association of Clinical Endocrinologists guidelines for man- agement of dyslipidemia and prevention of atherosclerosis. Endocr Pract. 2012;18 Suppl 1:1-78.

12. McCrindle BW, Urbina EM, Dennison BA, Jacobson MS, Steinberger J, Rocchini AP, et al. Drug therapy of high-risk lipid abnormalities in children and adolescents: a scientific statement from the American Heart Association Atherosclerosis, Hypertension, and Obesity in Youth Committee, Council of Cardiovascular Disease in the Young, with the Council on Cardiovascular Nursing. Circulation. 2007;115(14):1948-67.

13. Braamskamp MJ, Wijburg FA, Wiegman A. Drug therapy of hypercholesterolaemia in children and adolescents. Drugs. 2012;72(6):75972.

\section{CONFLITOS DE INTERESSE}

As autoras declaram não ter conflitos de interesse.

\section{ENDEREÇO PARA CORRESPONDÊNCIA}

Marisa Loio Rainho Rodrigues

Rua Prof. Carlos Alberto Mota Pinto, n. ${ }^{\circ} 91$

3100-492 Pombal

E-mail: mloio.rodrigues@gmail.com

Recebido em 07-02-2014

Aceite para publicação em 20-07-2014

\section{ABSTRACT}

\section{SCREENING FOR DYSLIPIDEMIAS IN CHILDREN AND ADOLESCENTS -THE EVIDENCE SUPPORTING THE RECOMMENDATIONS}

Recently, several medical societies have published clinical guidelines on screening for dyslipidemias in children and adolescents, including the Portuguese Directorate General of Health in June 2013. These recommendations have emerged in response to the increasing prevalence of overweight and obesity in childhood. Furthermore, studies have shown that the atherosclerotic process can begin in childhood and that children with dyslipidemias tend to maintain lipid profile disorders in adulthood. However, it is uncertain whether screening for dyslipidemias decreases cardiovascular risk, morbidity, and mortality.

This article reviews the different screening strategies proposed by the main medical societies, and analyzes the evidence supporting these recommendations.

Clinical guidelines on screening for dyslipidemias are not based on studies showing benefit in the reduction of cardiovascular risk, morbidity or mortality. The need to perform this screening should be considered for the individual patient.

Keywords: Dyslipidemias; Mass Screening; Child; Adolescent. 